Between October 2002 and September 2003, 1,476 patients with STEMI and 610 patients with non-STEMI were enrolled in the OPERA registry. The mean age of the population was 64 years, with $76 \%$ of participants being male.

During hospitalization, patients with STEMI were more likely to receive low-molecularweight heparin and were more likely to undergo percutaneous coronary intervention than were patients with non-STEMI ( $P<0.0001$ for both). By contrast, patients with non-STEMI were more likely to undergo CABG surgery $(P<0.05)$. In-hospital mortality rates were similar in the two groups. At hospital discharge, pharmacological secondary prevention was more aggressive in patients with STEMI than in patients with non-STEMI. After 1 year of follow-up, $36.7 \%$ of patients with STEMI and $41.5 \%$ of patients with non-STEMI had been rehospitalized for coronary disease. The proportion of patients requiring revascularization during follow-up was similar in the two groups. Multivariate analysis of the entire registry population identified history of heart failure, advanced age, untreated dyslipidemia and diabetes as independent predictors of 1-year post-discharge mortality. In subanalyses, the strongest predictors of death in both the STEMI and non-STEMI groups were history of heart failure and advanced age.

Original article Montalescot G et al. (2007) STEMI and NSTEMI: are they so different? 1 year outcomes in acute myocardial infarction as defined by the ESC/ACC definition (the OPERA registry). Eur Heart J 28: 1409-1417

\section{Heart failure in very elderly patients: clinical characteristics, treatment and outcomes}

Very elderly patients are often unrepresented in randomized controlled trials, and consequently do not always receive evidence-based therapies. Komajda and co-workers have analyzed data from the Euro Heart Failure Survey I, obtained at 115 centers in 24 countries, to assess the management of heart failure in patients aged $\geq 80$ years. In total, 2,780 octogenarians (median age 85.3 years) were compared with 7,912 younger patients (median age 69.0 years).

Both in-hospital and 12-week follow-up mortality were higher in the older patients than in the younger group (13.2\% vs $5.3 \%$, and $11.7 \%$ vs $6.1 \%$, respectively; $P<0.001$ for both). Octogenarians were significantly more likely than younger patients to have preserved left ventricular function, although fewer patients aged $\geq 80$ years had undergone echocardiography. The age-related differences in mortality were apparent in both patients with epressed and those with preserved systolic function. Comorbidities were more common in the older patient group, with only $13.1 \%$ of octogenarians free from comorbid conditions, compared with $22.4 \%$ of the patients aged $<80$ years $(P<0.001)$. Together with the presence of acute conditions, comorbidities were predictive of increased mortality, whereas use of angiotensin-convertingenzyme inhibitors, angiotensin-receptor blockers, antiplatelet agents or $\beta$-blockers was associated with improved outcomes. Importantly, the investigators found that angiotensin-convertingenzyme inhibitors and $\beta$-blockers were underused in octogenarians, suggesting that patient age might be deterring physicians from adhering to guidelines for prescription of these agents. By contrast, use of diuretics, digitalis and nitrates was more common in older than in younger patients.

Original article Komajda M et al. (2007) Management of octogenarians hospitalized for heart failure in Euro Heart Failure Survey I. Eur Heart J 28: 1310-1318

\section{Increased antithrombotic use puts the over $75 \mathrm{~s}$ at risk for stroke}

UK stroke mortality data indicate a decline in hemorrhagic stroke incidence over the past 20 years in individuals aged $<75$ years. Trends for intracerebral hemorrhage $(\mathrm{IH})$ in older individuals are unknown, but are likely to differ from those in younger people, as the predominant etiology of stroke alters with age. Lovelock et al. have compared data from the Oxford Community Stroke Project (OCSP; conducted 1981-1986) with data from the Oxford Vascular Study (OXVASC; 2002-2006) to examine changes in the incidence of $\mathrm{IH}$ over time.

The overall standardized incidence of all $\mathrm{IH}$ decreased over time. This decrease was, however, mostly attributable to a reduction in $\mathrm{IH}$ incidence in individuals aged $<75$ years; little change was noted in the incidence of $\mathrm{IH}$ in individuals aged $\geq 75$ years. The incidence of hypertensionassociated $\mathrm{IH}$ fell in both age-groups, but among 